American Journal of Animal and Veterinary Sciences 6 (4): 160-165, 2011

ISSN 1557-4555

(C) 2011 K. Mirhadi et al., This open access article is distributed under a Creative Commons Attribution

(CC-BY) 3.0 license

\title{
Effect of Intraperitoneally Injection of Different Doses of Lovastatin on Pain and Inflammatory Response Induced by Formalin in Mice
}

\author{
Khayatnouri Mirhadi \\ Department of Pharmacology, Tabriz Branch, Islamic Azad University, Tabriz, Iran
}

\begin{abstract}
Problem statement: The 3-Hydroxy-3-Methyl Glutaryl-CoA (HMG-CoA) reductase inhibitors (statins) have been unequivocally shown to reduce cardiovascular morbidity and mortality. Their lipid-lowering actions are by reversible and competitive inhibition of the enzyme HMG-CoA reductase, a precursor of cholesterol. It has been suggested that statins appear to have therapeutic benefits in diseases that are unrelated to elevated serum cholesterol levels, such as pain and inflammatory. The aim of this study was to determination the effect of intraperitoneally injection of different doses of lovastatin on pain and inflammatory response induced by formalin in mice. Approach: Mice were divided into 9 groups randomly: the first group received saline normal (ip) (saline group); the second group received Carboxymethylcellulose (CMC) $0.5 \%$ (ip) (vehicle group) and the next groups received respectively different doses of lovastatin $\left(1,5,10,20,40,80\right.$ and $\left.100 \mathrm{mg} \mathrm{kg} \mathrm{daily}^{-1}\right)$ for 4 days (ip) before formalin test. After intrapawly injection of formalin, the time of hind paw biting and licking time measured in 5 min interval for an hour. Results: Results showed that formalin induces biphasic pain response (the first phase: 0-5 min and the second phase 15-40 min after injection). Intraperitoneal injection of lovastatin showed significant $(p<0.05)$ decrease of pain responses in the first and the second phase in a dose dependently manner. Conclusion: According to our results, lovastatin has analgesic and anti-inflammatory effects on mice. Nevertheless, new studies must be carried out in order to determine the beneficial effects of statins in treatment of pain and inflammatory.
\end{abstract}

Key words: Nitric Oxide (NO), Tumor Necrosis Factor- $\alpha$ (TNF- $\alpha$ ), Rheumatoid Arthritis (RA), CReactive Protein (CRP), Heart Failure (HF), anti-inflammatory effect, centralized processing, integrated emotional phase, Collagen-Induced Arthritis (CIA)

\section{INTRODUCTION}

Pain is a percept consisting of initial nociception followed by a slower but integrated emotional phase. Nociception is the neural recognition of a potentially injurious physical or chemical stimulus. Pain responses occur only after centralized processing and elicitation of an emotional output to the nociceptive input. Longterm painful stimuli may enhance sensitivity in some animals and humans and habituate others. Acute centrally processed pain can be recognized in animals by behavioral changes (Kamerling, 1993; Ley and Livingston, 1990). The inflammatory response is an attempt by the body to restore and maintain homeostasis after injury or infection and is an integral part of body defense. For the survival of the host, inflammation is a necessary and beneficial process but chronic inflammation can cause harm (Beutler, 2003). Soluble mediators such as Nitric Oxide (NO), Tumor Necrosis Factor- $\alpha$ (TNF- $\alpha$ ) and interleukins usually play a role in controlling important functions such as the regulation of blood pressure, platelet aggregation and body temperature (Funk, 2001). Under pathologically inflammatory conditions, however, the production of these molecules promotes events ranging from increased leukocyte infiltration and vascular permeability to organ failure (Acquisto et al., 2002). The selective inhibition of these and other inflammatory activities remains an important goal for the effective treatment of inflammation.

The 3-Hydroxy-3-Methyl Glutaryl-CoA (HMGCoA) reductase inhibitors (statins) have been unequivocally shown to reduce cardiovascular morbidity and mortality (SSSSG, 1994; Shepherd et al., 1995). Statins are among the most widely used prescription drugs and exert their lipid-lowering actions by reversible and competitive inhibition of the enzyme 3-hydroxy-3-methyl-glutaryl-CoA reductase (HMGCoA reductase), the rate-limiting step in the conversion of 3-hydroxy-3-methylglutaryl-coenzyme A (HMGCoA) to mevalonate, a precursor of cholesterol (McTaggart et al., 2001). It has been suggested that statins appear to have therapeutic benefits in diseases that are unrelated to elevated serum cholesterol levels, 
such as rheumatologic diseases and ischemic stroke (McCarey et al., 2004). Statins are also known for their pleiotropic effects, which are independent of their lipidlowering properties. Among the effects of statins, the most relevant are anti atherosclerotic and antiinflammatory actions, improvement of endothelial dysfunction, anti-thrombosis and anti-oxidant actions, prevention of Alzheimer's disease and antineoplasic actions (Gonçalves et al., 2011). Although such clinical benefits are mediated in part through lipid modulation, recent studies demonstrate broader properties for statins, particularly in modifying inflammatory pathways ongoing within the atherosclerotic lesion (Ross, 1999). Statins might exert beneficial effects beyond cholesterol reduction; include improving endothelial function, decreasing vascular inflammation, inhibiting smooth-muscle proliferation and immunomodulation. Most of these effects are mediated through inhibition of isoprenoid synthesis, with subsequent effects on multiple downstream signaling pathways (Wang et al., 2008). Immunomodulatory activities of statins could be beneficial in treatment of various inflammatory conditions. This observation prompted subsequent in vitro studies that demonstrated that statins interfered with production of several important proinflammatory mediators (Pahan et al., 1997). Most of the reports point to the antiinflammatory effects of statins focused on cardiovascular diseases and atherosclerosis and about orally administration of these drugs (Garjani et al., 2008; Gonçalves et al., 2011; Kumar et al., 2010). Also studies showed that statins has a biphasic pro- and antiinflammatory effect (Garjani et al., 2008). Therefore the aim of this study was to determination of the effect of intraperitoneally injection of different doses of lovastatin on pain and inflammatory response induced by formalin in mice.

\section{MATERIALS AND METHODS}

Animals: Experiments were performed on 22-25 g adult NMRI male mice in their 8-9 week $(n=8$ for each group), purchased from Razi Institute (Iran). Animals were housed 8 per cage in the Animal House of Veterinary Faculty of Tabriz Branch, Islamic Azad University in a temperature $\left(20-22^{\circ} \mathrm{C}\right)$ and humidity $(50 \pm 10 \%)$ controlled environment under a $12 \mathrm{~h}$ light/dark cycle (lights on at $7 \mathrm{am}$ ). Food and water were available ad libitum. This study was performed in accordance with the Guide for the Care and Use of Laboratory Animals of Research affairs of Tabriz University of Medical Sciences, Tabriz-Iran. All efforts were made to minimize the number of animals which were used and their suffering degree. Animals were divided into 9 groups randomly: the first group received saline normal (ip) (saline group); the second group received Carboxymethylcellulose (CMC) $0.5 \%$ (ip) (vehicle group) and the next groups received respectively different doses of lovastatin $(1,5,10,20$, 40,80 and $100 \mathrm{mg} \mathrm{kg}$ daily $^{-1}$ ) for 4 days (ip) before formalin test.

Chemicals: Formalin was purchased from Merck Company. Lovastatin was provided from Merck Pharmaceuticals. All other reagents were of analytical grade. Lovastatin was prepared by being dissolved in 0.5\% Carboxy Methyl Cellulose (CMC) and the vehicle group was given an equal volume of vehicle.

Formalin test: Behavioral experiments were done in a quiet, temperature-controlled $\left(20-22^{\circ} \mathrm{C}\right)$ room between 10 am and $4 \mathrm{pm}$. Formalin test was preformed based on Dubission and Dennis method (Modified formalin test: characteristic biphasic pain response). After being weighted, the animals were placed in observation container for $15 \mathrm{~min}$ to get used to the new environment and then $20 \mu \mathrm{L}$ of attenuated formalin (5\%) was injected in hind paw skin (intradermal route) with insulin syringe in restrainer. After injection, animals were returned to observation container immediately and were viewed. The time of hind paw biting and licking time measured in $5 \mathrm{~min}$ interval for an $20 \mathrm{~h}$.

Data analysis: Group data are presented as mean \pm SEM and analyzed statistically using student test. Time course data were analyzed using one-way ANOVA followed by Tukey's post hoc test. The level for statistical significance was set at a $\mathrm{p}<0.05$.

\section{RESULTS}

Formalin injection to hind paw after intra peritoneal injection of normal saline induced significant $(\mathrm{p}<0.05)$ pain response (Time of licking and biting of injected paw) in the first, the fourth, the fifth, the sixth, the seventh and the $8-5 \mathrm{~min}$ in comparison with other 5 min (Fig. 1), as results show formalin induces biphasic pain response (the first phase: 0-5 min and the second phase 15-40 min after injection).

In vehicle group, CMC have not shown significant change on formalin induced biphasic pain response compared with saline group (Fig. 1). Intraperitoneal injection of lovastatin before hind paw injection of formalin showed significant $(p<0.05)$ decrease of pain responses (Time of licking and biting of injected paw), in the first phase (1-5 min) and the second phase (fourth, fifth, sixth, seventh and the 8-5 min) in a dose dependently manner compared with saline and vehicle groups (Fig. 1). 


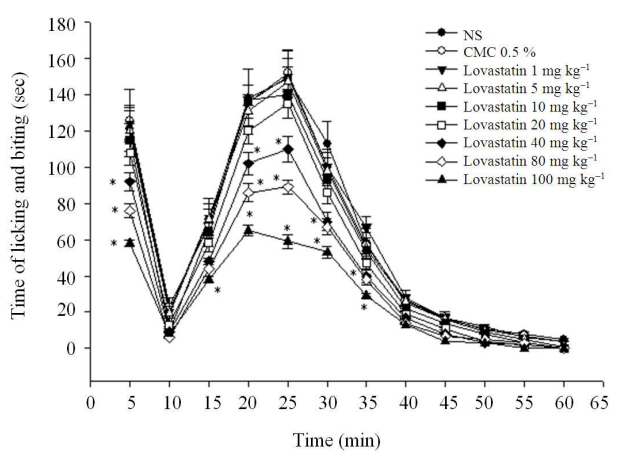

Fig. 1: Time course of paw licking and biting response to formalin injection. Effect of intraperitoneally administration of different doses of lovastatin on first and second phase of pain responses. Each line represents mean \pm SEM of 8 mice. $* \mathrm{p}<0.05$ compared with saline and vehicle groups

\section{DISCUSSION}

Formalin test has two phases; early or first phase (neurogenic pain) and delayed or second phase (inflammatory pain). It seems that early phase induced by $\mathrm{C}$ fiber activation and peripheral stimulates. But inflammatory reactions in peripheral tissues and action change in dorsal root of spine are the main reasons of delayed phase. The main reason of pain in the second phase is the inflammatory reactions (Abad et al., 2011). Results of present study have shown that lovastatin decreased pain and inflammatory response in formalin test, in a dose dependently manner, in both neurogenic and inflammatory origin.

Anti-nociceptive and anti-inflammatory effects of statins have been shown in different experimental animal models of pain and inflammatory and clinical studies, but effects of intraperitoneally injection of different doses of statins have not shown. Studies showed that lovastatin has a potent anti-inflammatory activity since it inhibits carrageenan-induced peritonitis and paw edema. Although these findings are consistent with studies showing these effects of statins in many inflammation models, most of them were carried out with other statins, mainly simvastatin (Dantas et al., 2010; Massaro et al., 2010; Nezic et al., 2009). Studies demonstrate that simvastatin provides a highly effective therapy in treating murine Collagen-Induced Arthritis (CIA), a surrogate model for human Rheumatoid Arthritis (RA). Previous studies of thioglycolate peritonitis or carrageenan-induced paw pad inflammation suggested that statins modify acute inflammation (Sparrow et al., 2001; Weitz-Schmidt et al., 2001). Histological examination of paw lesions confirmed that simvastatin inhibits the acute inflammation induced by carrageenan. These investigators concluded that simvastatin reduced the polymorphonuclear leukocyte infiltration, dosedependently and similarly to indomethacin (Nezic et al., 2009). Furthermore, atorvastatin was shown to be even more effective than diclofenac in decreasing joint inflammation and hyperalgesia in a rat model of arthritis (Mandosi et al., 2010) and in reducing TNF- $\alpha$ production in lipopolysaccharideactivated monocytes from diabetic patients (Trocha et al., 2010). In both experimental and clinical studies, statins have been shown to suppress acute and chronic inflammation. Their anti-inflammatory effect is brought about through inhibition of oedema formation, leukocyte-endothelial adhesion and production of inflammatory mediators (Kumar et al., 2010). Besides, they have been shown to afford protection in various experimental models of arthritis by reducing articular inflammation, preventing bone loss and preserving bone mineral density (Funk et al., 2008; Yamagata et al., 2007). Another statin, simvastatin, was reported to prevent the increase of iNOS concentration after ischemia/reperfusion injury in the rat liver. As well as in those studies, these effects of statins were mediated, at least in part, by the reduction of cytokines such as TNF- $\alpha$ and by the decrease of iNOS production. Besides being involved in inflammatory processes, iNOS has also been implicated in neuropathic pain. Hasegawa et al. (2003) reported that 6 weeks treatment with cerivastatin $\left(1 \mathrm{mg} \mathrm{kg} \mathrm{d}^{-1}\right)$ significantly attenuated the expression of pro-inflammatory cytokine interleukin 1b (IL-1b) in Dalt salt sensitive rats with left ventricular hypertrophy and chronic heart failure (Hasegawa et al., 2003). Lovastatin partially suppressed acute Experimental Autoimmune Encephalomyelitis (EAE) in rats (Stanislaus et al., 1999). Atorvastatin, one of the longer acting and clinically used statins, has been demonstrated to attenuate inflammatory hyperalgesia in a rat model of monoarticular arthritis. Atorvastatin has been documented to relieve hyperalgesia and to improve joint functions in the arthritic rats (Wahane and Kumar, 2010). Barsante et al. (2005) have reported anti-inflammatory and anti-hypernociceptive effects of atorvastatin in chronic arthritis induced by immunization of rats with adjuvant (Barsante et al., 2005). Clinical studies such as the West of Scotland Coronary Prevention Study (WOSCOPS), a randomized placebo controlled study, provided evidence that the anti-inflammatory effect of pravastatin treatment is 
probably independent of changes in the lipid profile (WOSCOPS, 1998). Statins have been used to reduce inflammation, tame immune cell activation, or arrest degenerative processes. Because of their widespread use and long-term safety record, some physicians prescribe statin therapy for non approved indications. A number of case reports describe the dramatic effects of statins added to standard therapies, but beneficial effects need to be confirmed in controlled studies. For example, in three patients with lupus glomerulonephritis who were not responding to the standard of therapy, adding high doses of simvastatin brought about a dramatic reduction in disease activity. There are randomized, placebo controlled studies, but two issues cloud the results: the particular statin used and the dose. Clearly the anti-inflammatory properties of statins vary, whereas the cholesterol-lowering properties are similar. Six clinically used statins were examined in vitro for their ability to affect NF-kB, phosphorylation of $\mathrm{IkB}$ and activation of tissue factor, the first step in coagulation. There was a distinct difference between the statins: cerivastatin, atorvastatin and simvastatin were more effective in reducing these parameters than fluvastatin, lovastatin, or pravastatin (Dinarello 2010). Also, other clinical studies demonstrated that in a small group of patients with Heart Failure (HF) and normal cholesterol levels that administration of atorvastatin $10 \mathrm{mg}$ for 4 weeks led to a significant reduction in IL-6, TNF-alpha and VCAM1 levels, that confirmed that the anti-inflammatory action of statin in this high risk group of patients (Tousoulis et al., 2005). Nevertheless, there seems to be no dearth of case reports and small uncontrolled trials that show the benefits of statins for reducing inflammation. In the case of rheumatoid arthritis where the anti-inflammatory effects of blocking cytokines have been shown repeatedly, there have been more than ten trials of statins resulting in a moderate reduction in joint inflammation associated with a fall in C-Reactive Protein (CRP) and red cell sedimentation rate and decreased cytokine production by circulating monocytes. As many patients with long-standing rheumatoid arthritis are also at high risk for cardiovascular disease, adding statin therapy to the standard of care is highly cost effective. Adding statins to the regimen of cyclosporine and sirolimus for kidney transplant patients lowered the rate of organ rejection. In patients with relapsing-remitting multiple sclerosis, statin therapy for 6 months significantly lowered the number of brain lesions detected by gadolinium, an established marker of disease activity. Examination of peripheral blood cells revealed no suppression in $\mathrm{T}$ cell responses but did reveal an increase in production of IL-10, an anti-inflammatory cytokine (Dinarello, 2010). Nevertheless, new and completely studies must be carried out in order to determine in more detail the beneficial actions of statins regarding the reduction of pro-inflammatory mediators.

\section{CONCLUSION}

According to our results, lovastatin has analgesic and anti-inflammatory effects on mice. Nevertheless, new studies must be carried out in order to determine the beneficial effects of statins in treatment of pain and inflammatory.

\section{REFERENCES}

Abad, A.N.A., M.H.K. Nouri, A. Gharjanie and F. Tavakoli, 2011. Effect of matricaria chamomilla hydroalcoholic extract on cisplatin-induced neuropathy in mice. Chinese J. Natural Med., 9: 126-131.

Acquisto, F.D., M.J. May and S. Ghosh, 2002. Inhibition of Nuclear Factor kappa B (NF-B): An Emerging theme in anti-inflammatory therapies. Mol. Interven, 2: 22-35. PMID: 14993359

Barsante, M.M., E. Roffe, C.M Yokoro, W.L. Tafuri and D.G. Souza, et al., 2005. Anti-inflammatory Anti-inflammatory and analgesic effects of atorvastatin in a rat model of adjuvant-induced arthritis. Eur. J. Pharmacol, 516: 282-289. DOI: 10.1016/j.ejphar.2005.05.005

Beutler, B., 2003. Innate immune responses to microbial poisons: Discovery and function of the toll-like receptors. Annu. Rev. Pharmacol Toxicol, 43: 609-628. DOI: 10.1146/annurev.pharmtox.43.100901.135729

Dantas, A.C., F.F. Batista-Junior, L.F. Macedo, M.N. Mendes and I.M. Azevedo et al., 2010. Protective effect of simvastatin in the cyclophosphamideinduced hemorrhagic cystitis in rats. Acta. Cir. Bras., 25: 43-46. PMID: 20126887

Dinarello, C.A., 2010. Anti-inflammatory agents: Present and future. Cell, 140: 935-950. DOI:10.1016/j.cell.2010.02.043

Funk, C.D., 2001. Prostaglandins and leukotrienes: Advances in eicosanoid biology. Science, 294: 1871-1875. DOI: $10.1126 /$ science.294.5548.1871

Funk, J.L., J. Chen, K.J. Downey and R.A. Clark, 2008. Bone protective effect of simvastatin in experimental arthritis. J. Rheumatol 35: 10831091. PMID: 18464303 
Garjani, A., S. Andabil, M. Ziaee and N. Maleki-dizaji, 2008. Biphasic effects of atorvastatin on inflammation. Pak. J. Pharm. Sci., 21: 125-130. PMID: 18390442

Gonçalves, D.O., I.B.F. Calou, R.P. Siqueira, A.A. Lopes and L.K.A. Leal et al., 2011. In vivo and in vitro anti-inflammatory and anti-nociceptive activities of lovastatin in rodents. Braz. J. Med. Biol. Res., 44: 173-181.

Hasegawa, H., R. Yamamoto H. Takano, M. Mizukami and M. Asakawa et al., 2003. 3-Hydroxy-3methylglutaryl coenzyme a reductase inhibitors prevent the development of cardiac hypertrophy and heart failure in rats. J. Mol. Cell Cardiol., 35: 953-960. DOI:10.1016/S0022-2828(03)00180-9

Kamerling, S.G., 1993. Narcotics and local anesthetics. Vet Clin North Am. Equine. Pract., 9: 605-620. PMID: 8299018

Kumar, V.L., B. Guruprasad and V.D. Wahane, 2010. Atorvastatin exhibits anti-inflammatory and antioxidant properties in adjuvant-induced monoarthritis. Inflammopharmacol, 18: 303-308. DOI: $10.1007 / \mathrm{s} 10787-010-0057-1$

Ley, S., A. Waterman and A. Livingston, 1990. Variation in the analgesic effects of xylazine in different breeds of sheep. Vet. Rec., 126: 508-508. PMID: 2368277

Mandosi, E., M. Fallarino, A. Gatti, A. Carnovale and M. Rossetti et al., 2010. Atorvastatin downregulates monocyte CD36 expression, nuclear NFkappaB and TNFalpha levels in type 2 diabetes. J. Atheroscler Thromb 17: 539-545. PMID: 20134099

Massaro, M., A. Zampolli, E. Scoditti, M.A. Carluccio and C. Storelli et al., 2010. Statins inhibit cyclooxygenase-2 and matrix metalloproteinase-9 in human endothelial cells: anti-angiogenic actions possibly contributing to plaque stability. Cardiovasc Res., 86: 311-320. DOI: 10.1093/cvr/cvp375

McCarey, D.W., I.B. Mclnnes, R. Madhok, R. Hampson and O. Scherbakov et al., 2004. Trial of Atorvastatin in Rheumatoid Arthritis (TARA): double-blind, randomised placebo-controlled trial. Lancet 363: 2015-2021. DOI: 10.1016/S01406736(04)16449-0

McTaggart, F., L. Buckett, R. Davidson, G. Holdgate and A. McCormick et al., 2001. Preclinical and clinical pharmacology of rosuvastatin, a new 3hydroxy- 3-methylglutaryl coenzyme A reductase inhibitor. Am. J. Cardiol 87: 28-32.
Nezic, L., R. Skrbic, S. Dobric, M.P. Stojiljkovic and S.S. Satara et al., 2009. Effect of simvastatin on proinflammatory cytokines production during lipopolysaccharide-induced inflammation in rats. Gen Physiol. Biophys., 28: 119-126. PMID: 19893089

Nezic, L., R. Skrbic, S. Dobric, M.P. Stojiljkovic and V. Jacevic et al., 2009. Simvastatin and indomethacin have similar anti-inflammatory activity in a rat model of acute local inflammation. Basic. Clin. Pharmacol Toxicol., 104: 185-191. DOI: $10.1111 / \mathrm{j} .1742-7843.2008 .00302 . \mathrm{x}$

Pahan, K., F.G. Sheikh, A.M. Namboodiri and I. Singh, 1997. Lovastatin and phenylacetate inhibit the induction of nitric oxide synthase and cytokines in rat primary astrocytes, microglia and macrophages. J. Clin. Invest., 100: 2671-2679. DOI: 10.1172/JCI119812

Ross, R., 1999. Atherosclerosis-an inflammatory disease. N. Engl. J. Med., 340: 115-126.

Shepherd, J., S.M. Cobbe, I. Ford, C.G. Isles and A.R. Lorimer et al., 1995. Prevention of coronary heart disease with pravastatin in men with hypercholesterolemia. West of Scotland Coronary Prevention Study Group. N. Engl. J. Med., 333:13011308.

Sparrow, C.P., C.A. Burton, M. Hernandez, S. Mundt and H. Hassing et al., 2001. Simvastatin has antiinflammatory and antiatherosclerotic activities independent of plasma cholesterol lowering. Arterioscler. Thromb. Vasc. Biol., 21: 115-121. DOI: 10.1161/01.ATV.21.1.115

SSSSG, 1994. Randomised trial of cholesterol lowering in 4444 patients with coronary heart disease: The Scandinavian Simvastatin Survival Study (4S). Lancet, 344: 1383-1389. DOI: 10.1016/S01406736(94)90566-5

Stanislaus, R., K. Pahan, A.K. Singh and I. Singh, 1999. Amelioration of experimental allergic encephalomyelitis in Lewis rats by lovastatin. Neurosci Lett., 269: 71-74. DOI: 10.1016/S03043940(99)00414-0

Tousoulis, D., C. Antoniades, E. Bosinakou, M. Kotsopoulou, and C. Pitsavos et al., 2005. Effects of atorvastatin on reactive hyperemia and inflammatory process in patients with congestive heart failure. Atherosclerosis 178: 359-363. DOI: 10.1016/j.atherosclerosis.2004.08.037

Trocha, M., A. Merwid-Lad, A. Szuba, E. Chlebda and M. Piesniewska et al., 2010. Effect of simvastatin on Nitric Oxide Synthases (eNOS, iNOS) and arginine and its derivatives (ADMA, SDMA) in ischemia/reperfusion injury in rat liver. Pharmacol. Rep., 62: 343-351. PMID: 20508290 
American J. Animal \& Vet. Sci., 6 (4): 160-165, 2011

Wahane, V.D. and V.L. Kumar, 2010. Atorvastatin ameliorates inflammatory hyperalgesia in rat model of monoarticular arthritis. Pharmacol. Res., 61: 329-333. DOI: 10.1016/j.phrs.2009.11.006

Wang, C.Y., P.Y. Liu and J.K. Liao, 2008. Pleiotropic effects of statin therapy: Molecular mechanisms and clinical results. Trends. Mol. Med., 14: 37-44. DOI: 10.1016/j.molmed.2007.11.004

Weitz-Schmidt, G., K. Welzenbach, V. Brinkmann, T. Kamata and J. Kallen, et al., 2001. Statins selectively inhibit leukocyte function antigen-1 by binding to a novel regulatory integrin site. Nat. Med., 7: 687-692. DOI: 10.1038/89058
WOSCOPS, 1998. Influence of pravastatin and plasma lipids on clinical events in the West of Scotland Coronary Prevention Study (WOSCOPS). Circulation, 97: 1440-1445. DOI: 10.1161/01.CIR.97.15.1440

Yamagata, T., K. Kinoshita, Y. Nozaki, M. Sugiyama and S. Ikoma et al., 2007. Effects of pravastatin in murine collagen-induced arthritis. Rheumatol. Int., 27: 631-639. DOI: 10.1007/s00296-006-0270-9 